Correlation studies revealed that disease activity (CRP, ESR, BASDAI) was associated to the alteration of a vast number of spliceosome components in the three subtypes of analyzed leukocytes. In addition, the BASFI index correlated with the expression of splicing factors ESRP1, SRSF1 and TRA2B in neutrophils. The inflammatory, atherogenic and oxidative profile of AS leukocytes was related to the expression of a number of spliceosome molecules in the three leukocyte subsets, although neutrophils exhibited the highest number of deregulated spliceosome components related to inflammation (STAT3, TNF- $\alpha$, IL- $1 \alpha$, IL-1 $\beta$, IL-5) and cell adhesion (ICAM-1, SELL, THBS4, CDH5). Finally, endothelial dysfunction correlated with the expression of several components of the major-spliceosome and splicing factors in monocytes and neutrophils.

Conclusions: 1) AS patients display a dysregulation of the splicing machinery components associated to disease function and activity. 2) The expression of spliceosome members in the different leukocyte subsets is related to the inflammatory, oxidative and proatherogenic profile of AS patients.

Alteration of the spliceosome may provide new biomarkers for diagnosis and clinical monitoring of AS

Acknowledgements: Funding by: JA PI-0314-2012, SER, ISCIII (RIER RD16/0012/0015)

Disclosure of Interest: None declared

DOI: 10.1136/annrheumdis-2017-eular.4845

\section{FRI0431 ALTERED EXPRESSION OF CIRCULATING MICRORNAS IS RELATED TO DISEASE ACTIVITY AND STRUCTURAL DAMAGE IN ANKYLOSING SPONDYLITIS PATIENTS}

P. Font, C. Perez-Sanchez, P. Ruiz-Limon, C. Lopez-Pedrera,

M.C. Castro-Villegas, M.C. Ábalos-Aguilera, N. Barbarroja, I. Arias-de la Rosa, M.D. Lopez-Montilla, A. Escudero-Contreras, C. Lopez-Medina,

E. Collantes-Estevez, Y. Jimenez-Gomez. Rheumatology Service, IMIBIC/Reina Sofia University Hospital/Cordoba University, Cordoba, Spain

Background: Ankylosing spondylitis (AS) remains difficult to diagnose before irreversible damage to sacroiliac joint is noticeable. MicroRNAs (miRNAs) are a group of single-stranded non-coding RNAs of 20-25 nucleotides in length that can potentially regulate every aspect of cellular function. Recent studies have demonstrated that miRNAs can be detected in the circulation and serve as potential biomarkers of various inflammatory pathologies.

Objectives: To evaluate whether AS pathogenesis is related to the aberrant expression of plasma miRNAs.

Methods: The expression profile of 800 miRNAs in plasma was determined in pooled samples from AS patients and healthy donors (HDs) ( $n=3$ each) using the nCounter technology. Next, candidate miRNAs were validated by real time RT-PCR in a cohort of AS patients and HDs ( $n=26$ each). In parallel, the expression of validated miRNAs was examined in a cohort of 24 psoriatic arthritis (PsA) patients. To evaluate their relevance in the AS pathogenesis, an analysis was performed by using the web-based bioinformatics tool Ingenuity Pathways Analysis (IPA). Association and correlation studies of altered miRNAs with clinical and analytical profile of the AS patients were also performed. AS activity was defined as CRP $>5 \mathrm{mg} / \mathrm{L}$ and/or BASDAI $>4$ and ESR $>20 \mathrm{~mm} / \mathrm{h}$. Structural damage was evaluated by mSASSS.

Results: In discovery phase, nine miRNAs were differentially expressed (fold change $\geq 2$ ) in the plasma of AS patients vs. HDs. After validation, higher expression levels of miRNA (miR)-146a-5p, miR-125a-5p, miR-151-3p and miR$22-3 p$, and lower of miR-150-5p and miR-451a were found in AS vs. HDs. Interestingly, higher miR-146a-5p, miR-125a-5p, miR-151-3p and miR-22-3p expression levels in AS than in PSA patients were further observed. The areas under the curve, generated to assess the accuracy of miRNAs as diagnostic biomarkers for AS, ranged from 0.717 to 0.798 ; the six-miRNAs signature reached 0.947 . Bioinformatics analysis revealed that miRNAs targeted inflammatory and bone remodeling genes, underlying their potential role in this pathology. Indeed, correlation studies showed an association between these six miRNAs and potential target proteins associated to AS pathophysiology. In addition, miR-146a$5 p$, miR-146a-5p, miR-125a-5p and miR-22-3p expression was increased in active vs. non-active patients. Moreover, miR-150-5p and miR-451a expression was related to the presence of syndesmophytes in AS patients, underlying their association with structural damage.

Conclusions: 1) Several miRNAs are found differentially expressed in plasma from AS patients vs. HDs and other chronic inflammatory pathologies as PsA, indicating that these miRNAs may be considered potential biomarkers for AS diagnose. 2) The differential expression of certain miRNAs is associated to disease activity and structural damage. Overall, this study identified a six-plasma
miRNAs signature that could be attractive candidates as non-invasive biomarkers for the AS diagnosis, and may help to elucidate the disease pathogenesis. Acknowledgements: Funded by JA PI-0314-2012, SER, and ISCIII (RIER RD16/0012/0015).

Disclosure of Interest: None declared

DOI: 10.1136/annrheumdis-2017-eular.4780

\section{FRIDAY, 16 JUNE 2017}

\section{Spondyloarthritis - clinical aspects (other than treatment)}

\section{FRI0430 DUAL TRAJECTORIES OF DISEASE ACTIVITY AND HEALTH-RELATED QUALITY OF LIFE IN PATIENTS WITH ANKYLOSING SPONDYLITIS}

A. van Tubergen ${ }^{1}$, M. Imkamp ${ }^{1,2}$, A. Boonen ${ }^{1}$, S. Arends ${ }^{3,4}$, M. Dougados $^{5}$ R. Landewé ${ }^{6}$, S. Ramiro ${ }^{7}$, F. van den Bosch ${ }^{8}$, D. van der Heijde ${ }^{7}$, F. Wink ${ }^{4}$ A. Spoorenberg ${ }^{3,4}$, V. Lima Passos ${ }^{2}{ }^{1}$ Maastricht University Medical Center ${ }^{2}$ University Maastricht, Maastricht; ${ }^{3}$ University Medical Center Groningen, Groningen; ${ }^{4}$ Medical Centre Leeuwarden, Leeuwarden, Netherlands; ${ }^{5}$ Hôpital Cochin, Paris, France; ${ }^{6}$ Amsterdam Medical Center, Amsterdam; ${ }^{7}$ Leiden University Medical Center, Leiden, Netherlands; ${ }^{8}$ Ghent University Hospital, Ghent, Belgium

Background: The ultimate goal of managing Ankylosing Spondylitis (AS) is to improve and maintain the patient's health-related quality of life (HRQoL). To reach this goal, rheumatologists target towards low disease activity, as this is the main modifiable factor. To date, there is insufficient insight into (1) the co-evolution of disease activity and HRQoL, and (2) the heterogeneity in such trajectories among patients.

Objectives: To explore the heterogeneous character of AS by identifying different temporal patterns of co-dependence between disease activity and HRQoL over 8 years follow-up.

Methods: Data from Outcome in AS International Study (OASIS; $n=161$ ) and Groningen Leeuwarden AS (GLAS; $n=264$ ) cohorts were used. All patients had an established diagnosis of AS. In GLAS, all patients started a biological. HRQoL and disease activity were measured bi-annually using ASQoL and ASDAS-CRP

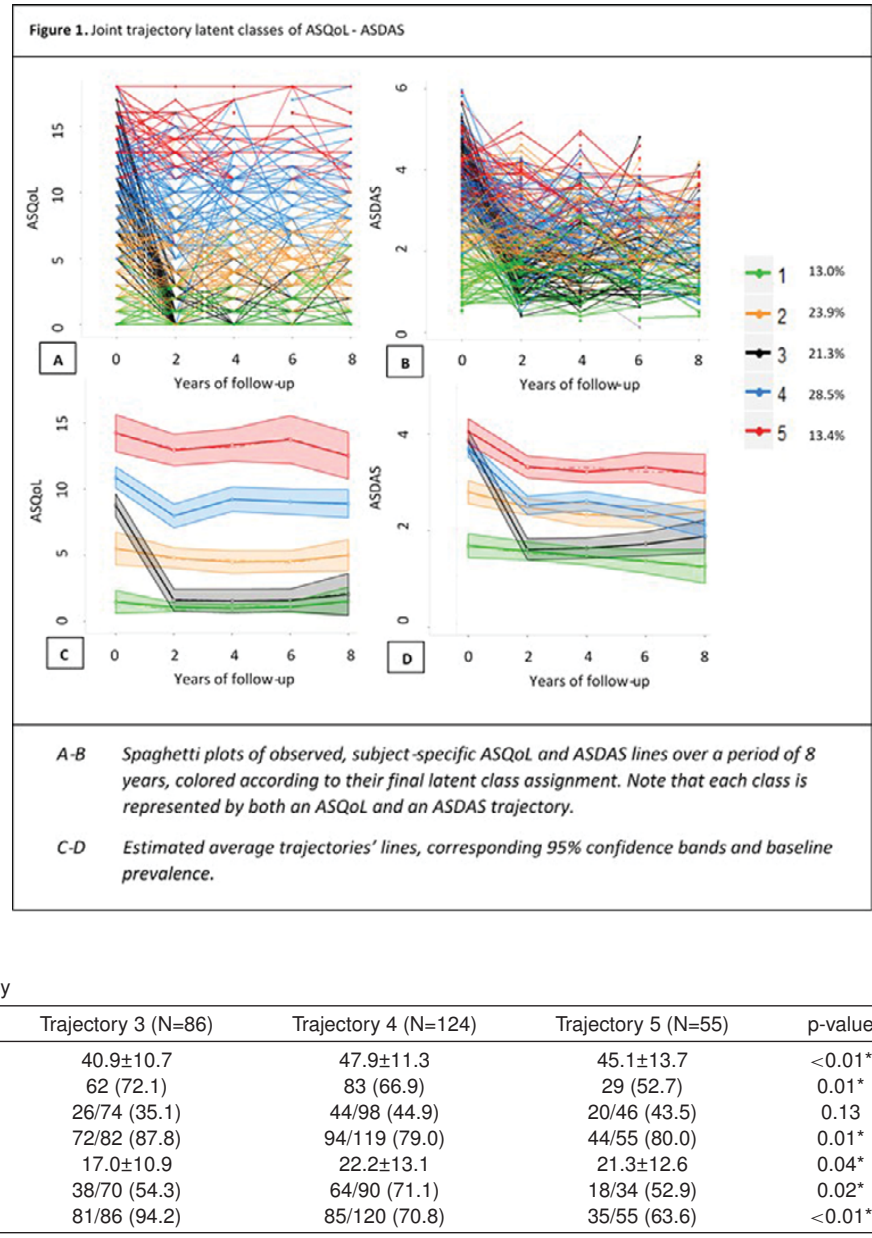

Abstract FRI0430 - Table 1. Baseline characteristics of patients within each ASQoL-ASDAS trajectory

\begin{tabular}{|c|c|c|c|c|c|c|}
\hline Characteristics & Trajectory $1(\mathrm{~N}=55)$ & Trajectory $2(\mathrm{~N}=105)$ & Trajectory $3(\mathrm{~N}=86)$ & Trajectory $4(\mathrm{~N}=124)$ & Trajectory $5(\mathrm{~N}=55)$ & p-value \\
\hline Age $-y r$ & $43.9 \pm 12.4$ & $46.0 \pm 12.7$ & $40.9 \pm 10.7$ & $47.9 \pm 11.3$ & $45.1 \pm 13.7$ & $<0.01^{\star}$ \\
\hline Male sex (\%) & $46(83.6)$ & $71(67.6)$ & $62(72.1)$ & $83(66.9)$ & $29(52.7)$ & $0.01^{*}$ \\
\hline Smoking - yes (\%) & $16 / 42(38.1)$ & $23 / 85(27.1)$ & $26 / 74(35.1)$ & $44 / 98(44.9)$ & $20 / 46(43.5)$ & 0.13 \\
\hline HLAB27 - positive (\%) & $50 / 54(92.6)$ & $73 / 103(70.9)$ & 72/82 (87.8) & $94 / 119(79.0)$ & $44 / 55(80.0)$ & $0.01^{*}$ \\
\hline Symptom duration $-\mathrm{yr}$ & $20.3 \pm 10.9$ & $21.4 \pm 12.1$ & $17.0 \pm 10.9$ & $22.2 \pm 13.1$ & $21.3 \pm 12.6$ & $0.04^{\star}$ \\
\hline (Bridging) syndesmophytes present (\%) & $16 / 39(41.0)$ & $45 / 73(61.6)$ & $38 / 70(54.3)$ & $64 / 90(71.1)$ & $18 / 34(52.9)$ & $0.02^{*}$ \\
\hline Start biological (\%) & $15 / 54(27.8)$ & $41 / 101(40.6)$ & $81 / 86(94.2)$ & $85 / 120(70.8)$ & $35 / 55(63.6)$ & $<0.01^{*}$ \\
\hline
\end{tabular}


respectively. Patients were classified into latent groups with individuals following a similar course of disease activity and HRQoL. These trajectories were estimated by Group-Based Trajectory Modelling. Next, the trajectories were profiled by comparing the latent groups with respect to baseline factors with ANOVA and Chi-square test.

Results: Five dual trajectories were revealed (Figure): 1. Low impact of AS on patient (13\%): stable low ASQoL and ASDAS inactive disease; 2. Moderate impact (24\%): stable moderate ASQoL and ASDAS high disease; 3 . Improving impact (21\%): major improvement in ASQoL and ASDAS; 4. High impact (29\%): moderately severe ASQoL with very high but improving ASDAS; 5 . Very High Impact (13\%): persistently severe ASQoL with high ASDAS. Low impact of AS was mainly characterized by male gender and HLA-B27; improving impact by younger age, short symptom duration, and biological intake; high impact by higher age, long symptom duration, and (bridging) syndesmophytes (Table).

Conclusions: We identified five dual trajectories of disease activity and HRQoL, each demonstrating a clear mutual relationship. These trajectories and their profiles provide insight into the heterogeneity of the impact of AS on patients' health and overall functioning.

Disclosure of Interest: None declared

DOI: 10.1136/annrheumdis-2017-eular.3802

\section{FRI0432 CLINICAL WORSENING ACCORDING TO THE PATIENT IS INFREQUENT IN AXIAL SPONDYLOARTHRITIS: RESULTS OF THE ASAS-FLARE STUDY IN 1169 PATIENTS}

$\underline{\text { A. Moltó }}^{1}$, B. Meghnathi ${ }^{1}$, L. Gossec ${ }^{2}$, R. Landewé ${ }^{3}$, D. van der Heijde ${ }^{4}$ M. Dougados ${ }^{1}$ on behalf of ASAS-FLARE study group. ${ }^{1}$ Paris Descartes University, Medicine Faculty; APHP, Rheumatology B Department, Cochin Hospital; ${ }^{2}$ Sorbonne Universités, UPMC Univ Paris 06; AP-HP, Pitié Salpêtrière Hospital, Department of rheumatology, Paris, France; ${ }^{3}$ Department of Clinical Immunology \& Rheumatology, Amsterdam Rheumatology Center, Amsterdam and Zuyderland Medical Center, Heerlen; ${ }^{4}$ Department of Rheumatology, Leiden University Medical Centre, Leiden, Netherlands

Background: Prevalence of flares/worsening of the disease in axSpA is not well known, with prevalences ranging from 10 to $40 \%$.

Objectives: To evaluate the prevalence of disease worsening according to the patient's perception in an axSpA population with stable disease and its correlation with disease activity parameters.

Methods: Study: International multicentric (20 countries) longitudinal (2 visits: 1 week - 6 months) observational in 2016, under the guidance of ASAS. Patients: axSpA patients with stable disease according to the rheumatologist. Data on disease characteristics were collected at baseline, and data on disease activity were collected at both visits. Disease worsening was defined at the follow-up visit by the patient using the MCID question ("Think about all the ways your spondyloarthritis has affected you during the last 48 hours. Compared to the last visit how did you feel during the last 48 hours? Improved/No change/Worse"). If patients answered "worse", they marked if they considered themselves in an acceptable symptoms state (PASS) and whether they considered treatment intensification was necessary. Analyses were descriptive and changes in disease activity were calculated according to patient-reported worsening

Results: Among the 1639 patients included, 1169 patients had complete data. Patients were predominantly males $(64.8 \%)$, had a mean age and disease duration of of 41.7 (SD 12.4) and 12.6 (9.9) years, respectively. History of X-ray sacroiliitis, MRI sacroiliitis and HLAB27+ were present in $944(80.8 \%)$, $471(40.6 \%)$ and $807(69.0 \%)$ patients, respectively. 56\% $(n=655)$ patients were receiving a biologic treatment. At the baseline visit, mean BASDAI (0-10) was 3.1 (2.3), mean ASDAS $2.3(1.0)$ and mean CRP $8.4 \mathrm{mg} / \mathrm{L}$ (14.5). Mean interval between both visits was $91.2(51.0)$ days. At the follow-up visit, $590(50.5 \%)$, $388(33.2 \%)$ and $191(16.3 \%)$ patients considered their condition had improved, not changed and worsened, respectively. Among the 191 patients reporting a worsening, $123(64.4 \%)$ considered their symptom status unacceptable, and 127 $(66.5 \%)$ judged their state required treatment intensification. BASDAI, ASDAS and CRP significantly increased in patients considering themselves worsening (Table).

\begin{tabular}{lccc}
\hline & Worsening & Not worsening & \\
\hline Change in BASDAI $(0-10)^{\star}$ & $1.3(1.8)$ & $-0.5(1.5)$ & $<0.005$ \\
Change in ASDAS* & $0.7(0.8)$ & $-0.3(0.8)$ & $<0.005$ \\
Change in CRP $(\mathrm{mg} /)^{\star}$ & $4.5(14.1)$ & $-2.0(13.2)$ & $<0.005$ \\
\hline
\end{tabular}

${ }^{*}$ Change is calculated as the absolute change between the visits; ${ }^{* \star}$ including improvement and no change in status.

Conclusions: in this real-life study of stable axSpA, worsening, as defined by the patient, was not frequent, but was significantly associated with increase in disease activity measures, including objective parameters such as CRP and not only patient-reported outcomes.

Acknowledgements: This study was supported by ASAS (Assessment in SpondyloArthritis international Society)

Disclosure of Interest: None declared

DOI: 10.1136/annrheumdis-2017-eular.4766

\section{FRI0433 HIGH PREVALENCE OF HIDRADENITIS SUPPURATIVA IN PATIENTS WITH AXIAL SPONDYLOARTHRITIS}

S. Arends ${ }^{1,2}$, A. Rondags ${ }^{3}$, F. Wink ${ }^{2}$, B. Horváth ${ }^{3}$, A. Spoorenberg ${ }^{1,2}$

${ }^{1}$ Rheumatology and Clinical Immunology, University Medical Center Groningen, Groningen; ${ }^{2}$ Rheumatology, Medical Center Leeuwarden, Leeuwarden;

${ }^{3}$ Dermatology, University Medical Center Groningen, Groningen, Netherlands

Background: Axial spondyloarthritis (SpA) is associated with several extraarticular manifestations such as the skin disease psoriasis. On the other hand, SpA was found to be more prevalent $(3-4 \%)$ in patients with another skin disease: hidradenitis suppurativa (HS). ${ }^{1} \mathrm{HS}$ is a chronic, recurrent, debilitating inflammatory skin disease that involves deep-seated painful nodules in the inverse body regions, with an average prevalence of $1 \%$ in the European population and a female predominance (ratio $3: 1) .^{2}$ Thus far, the prevalence of HS in axial SpA is not exactly known.

Objectives: To investigate the prevalence of HS in patients with axial SpA.

Methods: A self-screening questionnaire with validated questions concerning HS signs and symptoms including prototypical pictures was send to all participating patients from the Groningen Leeuwarden axial SpA (GLAS) cohort in 2016. All patients fulfilled the ASAS axial SpA criteria. Self-reported HS symptoms were verified by checking medical records and/or verification by phone, defined as diagnosis of HS by a dermatologist.

Results: In total, 588 questionnaires were send to the GLAS patients, of which 459 were returned and could be included in the final analysis (response rate $78 \%$ ). Of the included patients, mean age was $50 \pm 13$ years, $63 \%$ were male, mean symptom duration was $23 \pm 13$ years, and $78 \%$ were HLA-B27 positive.

The questionnaire data showed a high self-reported HS prevalence of $11 \%$. HS symptoms were confirmed by doctor's diagnosis in the large majority of these patients $(41 / 50 ; 82 \%)$, resulting in an estimated HS prevalence of $9 \%$.

The next step will be the comparison of patient characteristics and clinical assessments between axial SpA patients with and without HS.

Conclusions: The present observational cohort study shows that HS is a common skin disease in patients with axial SpA.

References:

[1] Richette et al. J Rheumatol 2014;41(3):490-4.

[2] Zouboulis et al. J Eur Acad Dermatol Venereol 2015;29(4):619-44

Disclosure of Interest: None declared

DOI: 10.1136/annrheumdis-2017-eular.6120

\section{FRI0434 POOR QUALITY OF LIFE IN PATIENTS WITH SPONDYLOARTHRITIS IS NOT EXPLAINED BY STRUCTURAL DAMAGE. DATA FROM REGISPONSER}

C. López-Medina, P. Font-Ugalde, J.L. Garrido-Castro, M.C. Castro-Villegas, J. Calvo-Gutiérrez, R. Ortega-Castro, A. Escudero-Contreras,

E. Collantes-Estévez on behalf of REGISPONSER working group.

Rheumatology, Hospital Universitario Reina Sofía de Córdoba/ Imibic/

Universidad de Córdoba, Córdoba, Spain

Background: In recent years it has become increasingly important the evaluation of the global impact of the disease in patients with Spondyloarthritis ( $\mathrm{SpA}$ ) through the use of the Patient-reported Outcomes (PROs) (1). One of the most used PROs is the Ankylosing Spondylitis Quality of Life (ASQoL) questionnaire, which refers to Health-Related Quality of Life (HRQoL). Since this is a subjective and multifactorial outcome (2), our goal is to detail the most important factors which are related with the Quality of Life $(\mathrm{QoL})$ in these patients.

Objectives: To evaluate QoL in patients with SpA and to define its association with disease-related factors and patient's features.

Methods: A cross-sectional multicenter study which includes 2229 patients with $\mathrm{SpA}$ selected from the national Spondyloarthropaties Spanish Registry (REGISPONSER). The main outcome was the assessment of QoL performed through the ASQoL questionnaire. Subsequently, we studied its relation with different factors organized into 5 groups: sociodemographics, emotional, functionality, disease-related factors and disease activity. Univariate logistic regressions and a multiple linear regression (considering ASQoL as a qualitative dichotomous and quantitative variable respectively) were performed to relate QoL with the studied covariates.

Results: The mean ASQoL score in the entire population studied was 6.09 \pm 5.12 . The average age was $47.74 \pm 13.26$ years old and $698(31.31 \%)$ were women. In univariate logistic regressions, significant differences $(p<0.05)$ were seen in many variables included in the 5 groups: poor QoL (ASQoL $\geq 9$ ) is related with gender (female), age, mental and physical component from SF-12 questionnaire, disease duration, inflammatory back pain (IBP), alternating buttock pain, BASRI (Bath Ankylosing Spondylitis Radiographic Index), BASFI (Bath Ankylosing Spondylitis Functional Index), BASDAI (Bath Ankylosing Spondylitis Disease Activity Index), ESR (Erythrocyte Sedimentation Rate) and global patient's VAS (Visual Analogue Scale), among others.

Finally, multivariate linear regression showed that $61.1 \%$ of the variability of ASQoL ( $\left.R^{2}=0.611, p<0.001\right)$ is explained by sex (female), physical component and 2nd item form SF-12 questionnaire (related to functionality), 6th and 7th items form SF-12 (both related to mental status), global patient's VAS, BASFI and BASDAI.

Conclusions: Poor QoL in SpA patients can be explained by high disease activity 